\title{
Estimativa do volume de Pinus taeda L. a partir de dados de sensoriamento remoto
}

\author{
Volume estimation from remote sensing information in a povoament of \\ Pinus taeda L.
}

\author{
Carla Talita Pertille ${ }^{1 *}$, Marcos Felipe Nicoletti ${ }^{1}$, Thiago Floriani Stepka ${ }^{1}$ e \\ Marcos Benedito Schimalski ${ }^{1}$
}

\begin{abstract}
Resumo
O objetivo desse trabalho foi estimar o volume de madeira de um plantio de Pinus taeda L. por meio da correlação entre dados espectrais e características dendrométricas do povoamento. Para o inventário florestal, foi utilizado processo de amostragem aleatória simples com método de área fixa utilizando-se parcelas circulares de $400 \mathrm{~m}^{2}$. A partir de imagens obtidas pelos sensores Landsat-8 e Sentinel-2, os índices de vegetação médios por parcela foram obtidos e correlacionados com o volume por parcela obtido pelo inventário florestal. Para os dados do Landsat-8, o índice com a melhor correlação foi o GNDVI com 0,5195 e para o Sentinel-2, o SAVI, com 0,4968. O ajuste das equações de regressão com esses índices apresentou $\mathrm{R}^{2}$ ajustado de 0,5145 e $\mathrm{S}_{\mathrm{yx}}$ (\%) de 14,74\% para o Landsat-8, e 0,4673 e 15,20\% para o Sentinel-2. Segundo a análise de variância, nấo houve diferença significativa entre o volume estimado pelo inventário florestal e por dados de Sensoriamento Remoto. Contudo, recomenda-se testar a metodologia aqui apresentada com imagens de alta resolução espacial e com outras espécies florestais.
\end{abstract}

Palavras chave: inventário florestal; índices de vegetação; reflectância.

\begin{abstract}
The objective of this work was to estimate the timber volume of a plantation of Pinus taeda L. by means of the correlation between spectral data and dendrometric characteristics of the stands. For the forest inventory, a simple random sampling process with fixed area method was used using circular plots of $400 \mathrm{~m}^{2}$. From the images obtained by the Landsat- 8 and Sentinel-2 sensors, the average vegetation indexes per plot were obtained and correlated with the volume per plot obtained by the forest inventory. For the Landsat- 8 data, the index with the best correlation was GNDVI with 0.5195 and Sentinel-2, SAVI, with 0.4968 . The adjustment of the regression equations with these indices showed adjusted $R^{2}$ of 0.5145 and Syx (\%) of $14.74 \%$ for Landsat-8, and 0.4673 and $15.20 \%$ for Sentinel-2. According to the analysis of variance, there was no significant difference between the volume estimated by the forest inventory and the Remote Sensing data. However, it is recommended to test the methodology presented here with high spatial resolution images and with other forest species.
\end{abstract}

Keywords: Forest inventory; vegetation indexes; reflectance.

\section{INTRODUÇÃO}

O volume de madeira é uma das variáveis mais importantes da floresta e seu monitoramento é uma das principais tarefas dos planos regionais de manejo florestal. A determinação do volume de madeira da floresta pode ser feita por métodos diretos e indiretos. O primeiro método compreende a mensuração como diâmetro em diferentes alturas e altura total, além da derrubada de árvores (SANQUETTA; BALBINOT, 2004) e pode ser um processo caro, com mão-de-obra intensiva (TESFAMICHAEL et al., 2010). A utilização do método indireto emprega equações alométricas ou ainda técnicas de Sensoriamento Remoto, para realização das suas estimativas (SANQUETTA; BALBINOT, 2004).

\footnotetext{
1. Departamento de Engenharia Floresta, Universidade do Estado de Santa Catarina - UDESC. Lages / SC, Brasil.

* Autor correspondente: carlatpertille@gmail.com
}

Sci. For., Piracicaba, v. 47, n. 123, p. 526-535, set. 2019 DOI: doi.org/10.18671/scifor.v47n123.14 
Os dados detectados remotamente contribuíram grandemente para avaliação e monitoramento florestal através da adição de um componente espacial à informação relacionada com a floresta. Este componente relaciona-se com a provisão de observação ou medição mais rápida e menos dispendiosa de muitos parâmetros de inventário florestal em diferentes áreas florestais. Além disso, métodos que empregam dados ópticos de alta resolução espacial e espectral fornecem estimativas razoavelmente precisas de várias características do inventário florestal, como alturas de dossel, volume de madeira, área basal e biomassa acima do solo (CHRYSAFIS et al., 2017).

A utilização de técnicas de Sensoriamento Remoto para a estimativa volumétrica de um povoamento florestal envolve a utilização de parcelas de inventário florestal (permanentes ou temporárias) instaladas em campo, para calibração de modelos preditivos. Nestes modelos, as diversas bandas espectrais dos sensores remotos, compreendem as variáveis preditoras, ao passo que o volume florestal será a variável resposta a ser estimada pixel a pixel (SOUSA et al., 2015).

A relação dos dados de campo com as imagens de satélite, pode ser obtida com o auxílio de índices de vegetação. Esses conforme Jensen (2009) são as combinações dos fatores de reflectância bidirecional - FRB de superfície, determinados em dois ou mais comprimentos de onda, associados a uma propriedade particular da vegetação, considerando as propriedades espectrais de folhas isoladas ou de dosséis vegetais.

Dentre os índices disponíveis na literatura, o primeiro índice desenvolvido foi o Razão Simples (SR - Simple Ratio) por Jordan (1969), sendo uma razão entre as bandas do infravermelho próximo e do vermelho. O Índice de Vegetação da Diferença Normalizada (NDVI - Normalized Difference Vegetation Index) é o mais conhecido e utilizado para estudos de caracterização e monitoramento da vegetação. Já o Índice de Vegetação Ajustado para o Solo (SAVI - Soil Adjusted Vegetation Index), proposto por Huete (1988) é caracterizado por reduzir os efeitos do solo de fundo no sinal da vegetação ao incorporar uma constante de ajuste de solo na equação. O Índice de Vegetação Verde da Diferença Normalizada (GNDVI - Green Normalized Difference Vegetation Index), proposto por Gitelson et al. (1996), evidencia a relação entre as bandas do infravermelho próximo e do verde e demonstra o aumento de sensibilidade em relação a clorofila. Outro índice indica relação significativa com as variáveis dendrométricas florestais é o Índice de Umidade da Vegetação (MVI - Moisture Vegetation Index), desenvolvido por Gao et al. (1996), o qual proporciona melhor contraste entre áreas secas e a vegetação, por ter sensibilidade as condições de umidade da vegetação.

Nesse sentido, como a avaliação do potencial produtivo de uma área florestal é realizada pelo inventário florestal, e, essa atividade demanda tempo e recursos, a utilização de dados remotamente situados surge como uma ferramenta eficiente e acessível na estimativa de variáveis dendrométricas, como o volume. Assim, a hipótese desse estudo é confirmar o potencial de utilização de imagens orbitais na extração de parâmetros florestais como o estoque volumétrico da floresta, com custo relativamente baixo e com a possibilidade de expandir tais estudos florestais, facilitando a gestão florestal.

Esse estudo teve como objetivo estimar o volume de madeira de um povoamento de Pinus taeda L., por meio da correlação entre valores de índices de vegetação obtidos pelos sensores remotos ópticos com as características dendrométricas do povoamento estudado.

\section{MATERIAL E MÉTODOS}

O estudo foi desenvolvido em um povoamento de Pinus taeda L. no município de Lages - Santa Catarina com talhões de aproximadamente 24 anos de idade. O clima da região é Cfb (clima mesotérmico subtropical úmido com verões frescos, sem estação seca e geadas severas), com temperatura média de $14,3^{\circ} \mathrm{C}$, umidade relativa do ar de $79,3 \%$ e precipitação bem distribuída ao longo do ano, com média anual de $1200 \mathrm{~mm}$ (ALVARES et al., 2013).

A área total de efetivo plantio conta com 24,58 hectares, distribuídas em oito talhões, as quais já passaram por intervenção de desbaste. O inventário florestal foi realizado utilizando o método de amostragem de área fixa e processo de amostragem aleatória simples. As 39 unidades amostrais instaladas foram circulares de $400 \mathrm{~m}^{2}$ e raio de 11,28 metros. O diâmetro à altura do peito (DAP) mensurado a 1,3 metros de todas as árvores existentes na parcela foi obtido com o auxílio da suta e a altura das dez primeiras árvores foram medidas com o Hipsômetro Blume-Leiss. Obteve-se a posição geográfica das parcelas com GPS (Global Positioning System) do modelo Garmin Etrex Legend ${ }^{\circledR}$ com 
precisão entre 10 a 15 metros, cujos dados foram georreferenciados no sistema de coordenadas UTM Datum WGS - 84 (World Geodetic System - 1984).

A cubagem rigorosa foi realizada pelo método de Smalian em 25 árvores divididas em diferentes classes de diâmetro, com diâmetro mínimo de $21 \mathrm{~cm}$ e máximo de 50,7 cm, 29,7 nas quais foram medidas as alturas dos tocos, as alturas totais e o diâmetro nas posições de 0,07m, 0,7 m, 1,30 m, 3,3 m, 5,3 m e assim sucessivamente de dois em dois metros até o final da árvore. Em seguida, ajustou-se o modelo não linear de Schumacher-Hall para a estimativa volumétrica individual.

A coleta de dados para aplicação das técnicas de Sensoriamento Remoto foi feita a partir dos sensores orbitais, Landsat-8 e Sentinel-2, descritos na Tabela 1:

Tabela 1. Caracterização dos sensores utilizados.

Table 1. Characterization of the sensors used.

\begin{tabular}{cccccc}
\hline Satélite & \multicolumn{5}{c}{ Resolução } \\
\cline { 2 - 6 } & Sensor & Espacial & Espectral & Radiométrica & Temporal \\
\hline Landsat-8 & OLI & $15 \mathrm{~m}$ e $30 \mathrm{~m}$ & 9 bandas & 16 bits & 16 dias \\
Sentinel-2 & $\mathrm{MSI}$ & $10 \mathrm{~m}, 20 \mathrm{~m} \mathrm{e} 60 \mathrm{~m}$ & 13 bandas & 12 bits & 5 dias \\
\hline
\end{tabular}

Onde: OLI (Operational Land Imager); MSI (Multispectral Instrument).

A etapa posterior compreendeu na avaliação da disponibilidade das imagens desses sensores nas datas em que foi realizado o inventário florestal na área. Além disso, outro critério para a aquisição das imagens foi a ausência ou pouca cobertura de nuvens.

A imagem do satélite Landsat-8 sensor OLI (Operational Land Imager) foi obtida na plataforma da United States Geological Survey, na órbita 221, ponto 79, com as bandas blue $(0,450-0,51 \mu \mathrm{m})$, green $(0,53-0,59 \mu \mathrm{m})$, red $(0,64-0,67 \mu \mathrm{m})$, near infrared $(0,85-0,88 \mu \mathrm{m}), \operatorname{SWIR} 1(1,57-1,65 \mu \mathrm{m})$, SWIR $2(2,11-2,29 \mu \mathrm{m})$ e PAN $(0,50-0,68 \mu \mathrm{m})$. A data da imagem é $10 / 09 / 2017$. O processamento digital dessa imagem foi realizado no aplicativo computacional ENVI (Environment for Visualizing Images), no qual foi realizada a correção atmosférica utilizando o algoritmo FLAASH (Fast Line-of-sight Atmospheric Analysis of Hypercubes).

A aquisição da imagem do Sentinel-2 sensor MSI foi realizada no portal Copernicus Open Acess $H u b$, na mesma órbita e ponto da outra imagem, para a data de 07/09/2017, nas seguintes bandas: blue $(490 \mu \mathrm{m})$, green $(560 \mu \mathrm{m})$, red $(665 \mu \mathrm{m})$, near infrared $(842 \mu \mathrm{m})$, Red-edge $1(705 \mu \mathrm{m})$, Red-edge 2 $(740 \mu \mathrm{m})$, Red-edge $3(785 \mu \mathrm{m})$, Red-edge $4(865 \mu \mathrm{m})$, SWIR $1(1610 \mu \mathrm{m})$ e SWIR $2(2190 \mu \mathrm{m})$. $\mathrm{O}$ processamento digital da imagem desse sensor foi realizado no aplicativo computacional Sentinel Application Platform (SNAP), no qual foi realizada a reamostragem das bandas (10 metros) e correção atmosférica utilizando a ferramenta Sen2Cor.

A resolução utilizada para cada sensor no cálculo dos índices foi de 30 metros para o Landsat-8/OLI e 10 metros para o Sentinel-2/MSI. Após o processamento das imagens, foram calculados os índices de vegetação, descritos na Tabela 2:

Tabela 2. Índices de vegetação calculados para o povoamento de Pinus taeda L. em Lages/SC.

Table 2. Indices of vegetation calculated for the settlement of Pinus taeda L. in Lages/SC.

\begin{tabular}{cc}
\hline IV & Equação \\
\hline GNDVI & $\frac{\rho \text { NIR- } \rho \text { GREEN }}{\rho \text { NIR }+\rho \text { GREEN }}$ \\
NDVI & $\frac{\rho \text { NIR- } \rho \text { SWIR }}{\rho \text { NIR }+\rho \text { SWIR }}$ \\
SAVI & $\frac{\rho \text { NIR- } \rho \text { RED }}{\rho \text { NIR }+\rho \text { RED }}$ \\
SR & $\frac{(1+L)(\rho \text { NIR- } \rho \text { RED })}{\rho \text { NIR }+\rho \text { RED }+L}$ \\
& $\frac{\rho \text { NIR }}{\rho \text { RED }}$
\end{tabular}

Onde: IV: Índice de Vegetação; GNDVI: Green Normalized Difference Vegetation Index; MVI: Moisture Vegetation Index; NDVI: Normalized Difference Vegetation Index; SAVI: Soil Adjusted Vegetation Index; SR: Simple Ratio Vegetation Index; $\rho$ GREEN: Reflectância da banda do verde; $\rho$ RED: Reflectância da banda do vermelho; $\rho$ NIR: Reflectância da banda do Infravermelho próximo; $\rho S W I R$ : Reflectância da banda do infravermelho de ondas curtas; L: constante que minimiza os efeitos do solo, utilizada nesse estudo o valor de 0,50 . 
O georreferenciamento da imagem foi realizado a partir dos pontos centrais da parcela obtidos pelo GPS. Com esses dados, a delimitação das parcelas nas imagens foi feita utilizando a ferramenta buffer, a qual permitiu construir uma área igual ao raio da parcela, de 11,28 metros. A relação entre a área da parcela e a área do pixel variou para cada sensor utilizado, sendo que para o Landsat-8/OLI a grande maioria das parcelas estava em um pixel $\left(900 \mathrm{~m}^{2}\right)$ enquanto que para o Sentinel-2/MSI as parcelas ocuparam entre 2 a 4 pixels $\left(100 \mathrm{~m}^{2}\right)$. Posteriormente, foram obtidos os valores médios dos índices de vegetação por parcela utilizando a ferramenta Zonal Statistics as a Table em ambiente SIG (ESRI, 2017).

A análise estatística inicial compreendeu a análise de correlação de Pearson entre o volume de madeira obtido na parcela $\left(\mathrm{m}^{3} / 0,04 \mathrm{ha}\right)$ e os valores médios dos índices de vegetação por parcela. Para a definição dos dados orbitais que propiciam a melhor estimativa do volume de madeira, utilizou-se análise de regressão. As variáveis obtidas nas imagens foram consideradas como independentes, enquanto que os dados oriundos do inventário florestal foram as variáveis dependentes. $\mathrm{O}$ índice de vegetação com a melhor correlação foi utilizado no ajuste dos modelos de regressão (Tabela 3 ) afim de estimar o volume da parcela $\left(\mathrm{m}^{3} / 0,04 \mathrm{ha}\right)$.

Tabela 3. Modelos ajustados a partir dos índices de vegetação melhor correlacionados com o volume para a estimativa do volume por parcela ( $\left.\mathrm{m}^{3} / 0,04 \mathrm{ha}\right)$.

Table 3. Models adjusted from vegetation indexes best correlated with volume for volume estimation by plot $\left(\mathrm{m}^{3} / 0,04 \mathrm{ha}\right)$.

\begin{tabular}{ccc}
\hline Modelo & \multicolumn{1}{c}{ Equação } & Autor \\
\hline 1 & $\mathrm{~V}=\beta 0^{*} \mathrm{IV}^{\beta 1}+\varepsilon$ & Berkhout \\
2 & $\mathrm{~V}=\beta 0^{*} \beta 1^{*} \frac{1}{\mathrm{IV}}+\varepsilon$ & Curtis \\
3 & $\mathrm{~V}=\beta 1^{*} \mathrm{IV}+\beta 2 * \mathrm{IV}^{2}+\varepsilon$ & Dissescu-Meyer \\
4 & $\mathrm{~V}=\beta 0+\beta 1^{*} \mathrm{IV}+\beta 2 * \mathrm{IV}^{2}+\varepsilon$ & Hohenadl-Krenm \\
5 & $\mathrm{~V}=\beta 0+\beta 1^{*} \mathrm{IV}^{2}+\varepsilon$ & Kopezky-Gehrardt \\
\hline $\begin{array}{l}\text { Onde: } \mathrm{V}: \text { volume por parcela }\left(\mathrm{m}^{3} / 0,04 h \mathrm{~h}\right) ; \beta ; \\
\text { de vegetação; } \text { : } \text { erro associado ao modelo. }\end{array}$
\end{tabular}

A escolha da melhor equação ajustada atendeu aos critérios estatísticos de maior coeficiente de determinação ajustado ( $\mathrm{R}^{2}$ aj.) e menores valores de erro padrão da estimativa (Syx\%) de acordo com Nicoletti et al. (2016).

Os dados obtidos pela regressão e pelo SR foram comparados e submetidos ao teste de normalidade pelo teste de Shapiro-Wilk. O delineamento foi o inteiramente casualizado com três tratamentos (volume predito pelo inventário florestal, volume estimado pelo índice de vegetação GNDVI para o Landsat-8 e volume estimado pelo índice de vegetação SAVI para o Sentinel-2). Foi realizada a Análise de Variância (ANOVA) para avaliar a significância entre os métodos. Em caso de diferenças significativas, aplica-se o teste de médias de Tukey para verificar as diferenças entre os tratamentos. As análises foram realizadas no software R versão 3.4.1. (R CORE TEAM, 2018). E, por fim, foi possível realizar o mapeamento do volume de madeira por talhão para o povoamento de Pinus taeda L. em ambiente SIG (ESRI, 2017).

\section{RESULTADOS E DISCUSSÃO}

Com os dados do inventário florestal, ajustou-se o modelo não linear de Schumacher-Hall para a estimativa volumétrica individual, com os parâmetros do modelo representados na Equação 1. As estatísticas do ajuste revelaram que, com base no valor de p, os parâmetros da equação foram significativos a 5\%, com $\mathrm{R}^{2}$ ajustado de 0,9568 e erro padrão da estimativa de 6,06\%.

$$
\mathrm{v}=-3,22701+\operatorname{dap}^{0,31053 * \mathrm{~h}^{0,10748}}
$$

Em que: $\mathrm{v}=$ volume individual estimado $\left(\mathrm{m}^{3}\right) ; \mathrm{h}=$ altura total $(\mathrm{m})$; dap = diâmetro à altura do peito $(\mathrm{cm})$. 
As características das variáveis dendrométricas para o povoamento de Pinus taeda L. foram: DAP médio: 3,6 cm; área basal por hectare: 79,1 $\mathrm{m}^{2}$ e altura total média de 22,1 metros. Os diâmetros mínimo e máximo do povoamento variaram de $21 \mathrm{~cm}$ à $50,7 \mathrm{~cm}$ respectivamente. Já as alturas variaram entre $15 \mathrm{~m}$ a $28 \mathrm{~m}$. O volume predito pelo inventário florestal variou de $11,8 \mathrm{~m}^{3} / 0,04 \mathrm{ha}$ a $28,7 \mathrm{~m}^{3} / 0,04 \mathrm{ha}$, com média de $20,8 \mathrm{~m}^{3} / 0,04 \mathrm{~h}$. Já o número de árvores por hectare foi de 378,1 .

A análise de correlação entre os índices de vegetação médios por parcela com o volume por parcela (Tabela 4) revelou correlação positiva significativa entre os mesmos. Os índices oriundos do Landsat-8 variaram entre 0,0499 e 0,5195, enquanto que os dados do Sentinel-2 apresentaram pouca variação. Além disso, com exceção dos índices GNDVI e SR, os índices obtidos a partir do Landsat-8 foram menores dos índices derivados do Sentinel-2. A maior correlação para o sensor Landsat-8 foi observada no índice GNDVI, com 0,5195 enquanto que para os dados do Sentinel-2, o índice que apresentou a maior correlação foi o SAVI, com 0,4968.

Tabela 4. Coeficientes de correlação de Pearson entre volume por parcela (dependente) e os índices de vegetação médios por parcela obtidos com o Landsat-8 e Sentinel-2 (independentes) para um povoamento de Pinus taeda L. em Lages-SC.

Table 4. Pearson correlation coefficients between volume per plot (dependent) and mean vegetation indices per plot obtained with Landsat-8 and Sentinel-2 (independent) for a stand of Pinus taeda L. in Lages-SC.

\begin{tabular}{cccc}
\hline Landsat-8 & Volume & Sentinel-2 & Volume \\
\hline Volume & 1 & Volume & 1 \\
NDVI & $0,2953^{*}$ & NDVI & $0,4461^{*}$ \\
SAVI & $0,3024^{*}$ & SAVI & $0,4968^{*}$ \\
GNDVI & $0,5195^{*}$ & GNDVI & $0,4508^{*}$ \\
MVI & $0,0499^{*}$ & MVI & $0,4740^{*}$ \\
SR & $0,4427^{*}$ & SR & $0,4839^{*}$ \\
\hline
\end{tabular}

*Correlação significativa a $5 \%$ de probabilidade.

Os valores de correlação obtidos para os dois sensores avaliados podem ser explicados em função dos índices de vegetação testados utilizarem bandas espectrais na região do visível (especialmente o vermelho) e do infravermelho. O comportamento espectral da vegetação nesses intervalos espectrais apresenta um antagonismo: no visível ocorre forte absorção e no infravermelho, máxima reflectância. Além disso, uma característica do povoamento também influenciou nessa análise, a idade. Como o povoamento possui 24 anos, o dossel, em sua grande parte, está em sua grande parte fechado, mesmo com a ocorrência de desbastes. Assim, a correlação positiva encontrada corrobora com a afirmação de Spanner et al. (1990) de que, dosséis fechados apresentam correlações positivas e correlações negativas são encontradas em áreas com dosséis abertos.

As características do povoamento como altura, tipo de manejo e o relevo da área podem ter influenciado na relação existente entre os índices de vegetação e o volume. A influência desses fatores foi destacada por Berra et al. (2016), que associam a relação inversa entre a faixa do infravermelho e o volume com a presença de sombreamento e formação de estratos em função das alturas homogêneas.

Já o ajuste dos modelos de regressão visando estimar o volume por parcela a partir dos índices de vegetação com a maior correlação (GNDVI e SAVI) demonstrou estatísticas de ajuste relativamente baixas, com coeficiente de determinação ajustado inferior a 50\% (exceto para os modelos de Berkout e Dissescu-Meyer) e erro padrão da estimativa de aproximadamente 15\% (Tabela 5).

A análise gráfica dos resíduos para os modelos (Figura 1) ilustra um comportamento semelhante dos resíduos em praticamente todos os modelos ajustados. É possível notar a ausência de independência dos erros, um dos pressupostos da análise de regressão em todos os modelos. Além disso, houve subestimativas e super estimativas, bem como a presença de outliers. Entretanto, o modelo que apresentou a melhor estimativa de volume em função dos índices de vegetação melhores correlacionados para os dados do Landsat-8 e Sentinel-2 foi o modelo de Berkout, em função do maior coeficiente de determinação ajustado $(0,5145$ e 0,4673) e menor erro padrão da estimativa $(14,74 \%$ e $15,20 \%$, respectivamente) e da melhor distribuição gráfica dos resíduos.

A qualidade do ajuste do modelo de Berkout para a estimativa do volume por parcela ( $\left.\mathrm{m}^{3} / 0,04 \mathrm{ha}\right)$ pode ser explicada pela idade do povoamento e pelas características do dossel, como geometria e reflectância das folhas Outro fator decisivo foi a iluminação no povoamento, pois a interferência 
Tabela 5. Estatísticas de ajuste dos modelos de regressão testados para os sensores Landsat- 8 e Sentinel-2 visando estimar o volume por parcela ( $\mathrm{m}^{3} / 0,04 \mathrm{ha}$ ) para um povoamento de Pinus taeda L. em Lages - SC.

Table 5. Adjustment statistics of the regression models tested for the Landsat- 8 and Sentinel-2 sensors to estimate the volume per plot ( $\left.\mathrm{m}^{3} / 0,04 \mathrm{ha}\right)$ for a Pinus taeda L. settlement in Lages-SC.

\begin{tabular}{cccccc}
\hline \multirow{2}{*}{ Modelo } & \multicolumn{5}{c}{ Landsat-8 } \\
\cline { 2 - 6 } & $\beta_{0}{ }^{*}$ & $\beta_{1}{ }^{*}$ & $\beta_{2}{ }^{*}$ & R2aj & Syx (\%) \\
\hline 1 & 39,36631 & 0,38552 & - & 0,5145 & 14,7 \\
2 & 26,63334 & $-1,08190$ & - & 0,2110 & 15,0 \\
3 & - & 186,01079 & $-392,02053$ & 0,9514 & 15,0 \\
4 & 12,57132 & 38,42191 & 20,47511 & 0,2294 & 14,8 \\
5 & 15,76811 & 129,67082 & - & 0,2477 & 14,7 \\
\hline & \multicolumn{7}{c}{ Sentinel-2 } & 0,4673 & 15,2 \\
2 & 23,84563 & 0,30784 & - & 0,1275 & 15,8 \\
4 & 23,58902 & $-1,75496$ & - & 0,9463 & 16,7 \\
5 & - & 58,42262 & $-39,54306$ & 0,2359 & 14,8 \\
\end{tabular}

Onde: $\beta_{i}^{*}$ : parâmetros do modelo com significância a $5 \%$ de probabilidade; $R^{2}$ aj: coeficiente de determinação ajustado; Syx: erro padrão da estimativa em percentagem (\%).

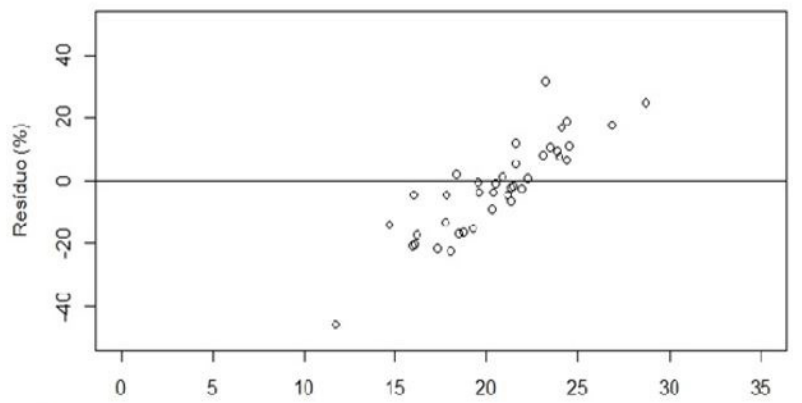

A)

Volume ( $\left.m^{3} / 0,04 h a\right)$

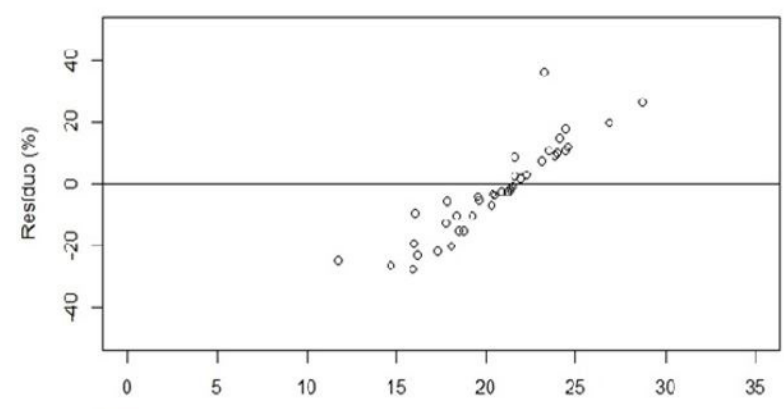

B)

Figura 1. Dispersão gráfica dos resíduos para o melhor modelo ajustado com os dados do Landsat-8 (A) e com os dados do Sentinel-2 (B) na estimativa de volume por parcela $\left(\mathrm{m}^{3} / 0,04 \mathrm{ha}\right)$ de um povoamento de Pinus taeda L. em Lages - SC.

Figure 1. Graphical dispersion of the residues for the best model fitted with the Landsat- $8(\mathrm{~A})$ data and the Sentinel-2 (B) data in the volume estimate per plot (m3 / 0.04ha) of a settlement of Pinus taeda L in Lages - SC.

dessa condição aliada com a topografia do local, em diferentes comprimentos de onda, influenciou nos valores de refletância bidirecional de superfície e, consequentemente, nos valores dos índices de vegetação obtidos (CANAVESI et al., 2010).

Apesar da qualidade do ajuste dos modelos de regressão, a análise da Tabela 6 permite afirmar que a média do volume por talhão estimado pelos índices de vegetação estimou de maneira satisfatória o volume em relação ao método tradicional de inventário florestal. Já o mapa do volume correspondente a cada talhão foi realizado com base no volume obtido pelo inventário florestal, como demonstra a Figura 2.

Tabela 6. Estimativa do volume por parcela ( $\mathrm{m}^{3} / 0,04 \mathrm{ha}$ ) obtidas pelo inventário florestal e pelo ajuste dos modelos de regressão utilizando os índices de vegetação oriundos do Landsat-8 e Sentinel-2.

Table 6. Estimation of the volume per plot $\left(\mathrm{m}^{3} / 0,04 \mathrm{ha}\right)$ obtained from the forest inventory and the adjustment of the regression models using the vegetation indexes from Landsat- 8 and Sentinel- 2 .

\begin{tabular}{cccc}
\hline Talhão & Inventário & Landsat-8 & Sentinel-2 \\
\hline 1 & 16,7 & 17,7 & 18,0 \\
2 & 22,8 & 21,4 & 21,3 \\
3 & 24,0 & 20,3 & 20,6 \\
4 & 20,6 & 21,8 & 21,6 \\
5 & 20,3 & 21,3 & 21,2 \\
6 & 20,4 & 21,0 & 20,9 \\
Média (m³/0,04ha) & 20,8 & 20,6 & 20,6 \\
\hline
\end{tabular}




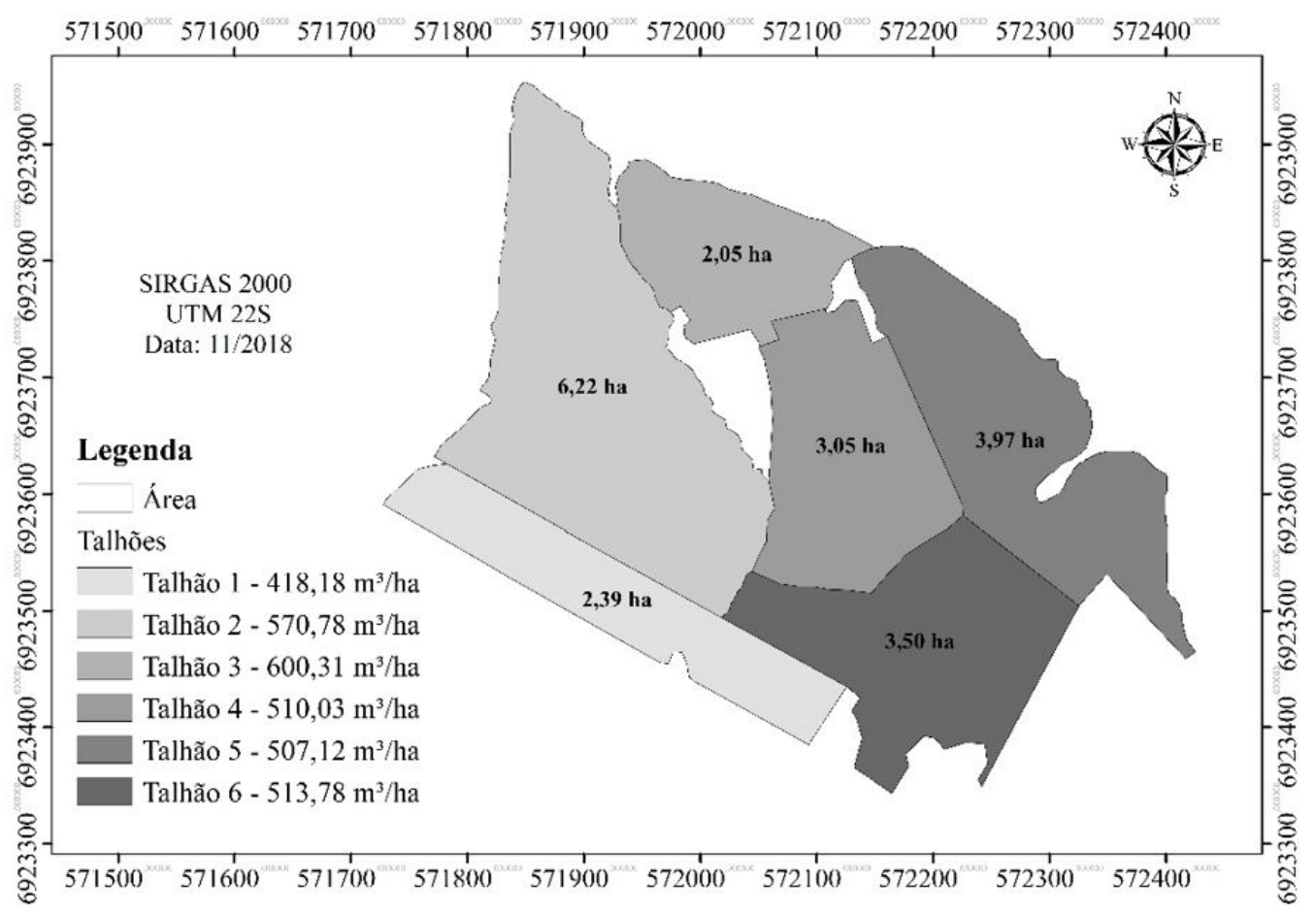

Figura 2. Mapa do volume correspondente a área de cada talhão derivado do inventário florestal para o povoamento de Pinus taeda L. em Lages - SC.

Figure 2. Volume map corresponding to the area of each plot derived from the forest inventory for the settlement of Pinus taeda L. in Lages - SC.

O teste de Shapiro-Wilk revelou que os dados seguem distribuição normal e por isso, aplicou-se a análise da variância. Tal análise estatística também comprovou a igualdade entre os métodos, sendo que, a análise de variância indicou que não existem diferenças entre as médias do volume estimado pelo inventário florestal e do volume obtido com os índices de vegetação para os sensores Landsat- 8 e Sentinel-2.

De maneira geral, tanto o Landsat- 8 como o Sentinel-2 demonstraram sensibilidade as mudanças do volume de madeira. O aumento da variável volumétrica ocasionou a redução dos valores de reflectância nas bandas do visível e no infravermelho enquanto que na faixa espectral do infravermelho médio e nos valores dos índices de vegetação essa variável aumentou.

Os dados remotamente situados oriundos do sensor Landsat-8 apresentaram correlação significativa com o GNDVI, o que pode ser explicado pela substituição da banda vermelha pela banda verde no cálculo desse índice e pelo comportamento explicado anteriormente da reflectância nessas bandas espectrais.

$\mathrm{Na}$ literatura consultada, grande parte das pesquisas com sensores Landsat foram para florestas de eucalipto (ALBA et al., 2017; LEAL et al., 2013; SANTOS et al., 2017).

Apenas o estudo de Berra et al. (2016) retratou a estimativa de volume de um povoamento de Pinus elliotti Engelm. a partir de dados Landsat-5 TM e LISS-III/ResourceSat-1. Nesse trabalho, os resultados da correlação linear obtidos com os dados do Landsat- 5 foram inferiores aos adquiridos com LISS-III. Contudo, os valores de reflectância espectral em quatro faixas espectrais (azul, verde, vermelho e infravermelho) foram equivalentes para os dois sensores que representaram sensibilidade as alterações do potencial volumétrico da área, indicando possibilidade de integração entre imagens LISS e TM para tais pesquisas.

Dentre os trabalhos citados, Leal et al. (2013) avaliaram a utilização de índices de vegetação como fator de correção do volume para estimativa volumétrica de um povoamento florestal de eucalipto no município de Rio Verde, Goiás. Os resultados indicaram baixa correlação entre os índices de vegetação e o volume obtido com o inventário, sendo que o NDVI apresentou a melhor correlação com a variável volumétrica. Entre os modelos de regressão testados, o que apresentou o melhor desempenho foi o de Berkout, com $\mathrm{R}^{2}$ ajustado de 0,54 e erro padrão da estimativa de 13,10\%. Os autores concluíram que tal abordagem não gerou estimativas precisas do estoque de madeira do povoamento avaliado e atribuíram esse fato a diferença dos procedimentos testados. 
Alba et al. (2017) avaliaram a identificação de plantios comerciais de Eucalyptus grandis de diferentes idades e a estimativa do volume total de madeira dessas áreas utilizando imagens do sensor OLI/Landsat-8. Os dados remotamente situados permitiram a diferenciação dos estágios de crescimento da espécie em questão e a estimativa do volume através do índice SAVI, que melhor representou as alterações do volume do povoamento. A seleção das variáveis pelo método Stepwise gerou modelos ajustados com $\mathrm{R}^{2}$ ajustados superiores a 0,70 e erro padrão da estimativa de $60,08 \mathrm{~m}^{3} / \mathrm{ha}$.

Já Santos et al. (2017) investigaram a viabilidade da utilização de imagens multiespectrais do sensor Landsat-8/OLI através de análise de regressão com dados de campo, para a estimativa de parâmetros florestais em área de cerrado. Para o volume, o $\mathrm{R}^{2}$ ajustado variou de 0,40 a 0,49. Os autores concluíram que essa metodologia apresenta potencial de aplicação em áreas do Cerrado e que o NDVI apresentou a melhor correlação com as variáveis estimadas.

Reuveni et al. (2018) utilizaram combinação de bandas do Landsat-7 sensor ETM+ (Thematic Enhanced Thema Plus) na estimativa de variáveis florestais para a floresta Lahav e a floresta Kramin, no norte israelense. Os autores utilizaram elementos de estruturação morfológica, algoritmos de combinação convexa e algoritmo de combinação de banda espectrais, para obter a melhor correlação entre o inventário florestal e os conjuntos de combinações lineares das bandas espectrais Landsat-7 ETM+. Com essa metodologia, o erro médio quadrático obtido foi de 1,48 $\mathrm{m}^{3} /$ ha e $6,31 \mathrm{~m}^{3} /$ ha, que sugerem a aplicação desse procedimento para outras florestas e dados de satélites.

A utilização do Sentinel-2 para estimativa de parâmetros florestais ainda é recente, porém já demonstra resultados satisfatórios e grande potencial de utilização, como é possível perceber na pesquisa de Nink et al. (2015). Ao avaliar a integração de avaliações de recursos florestais e informações espectrais para estimar a distribuição espacial do volume de madeira nas florestas de abeto da Noruega (Picea abies) a partir de dados dos sensores Sentinel-2/MSI e EnMAP, os autores concluíram que a capacidade espectral e espacial do Sentinel-2/MSI (inclusão das bandas conhecidas como red-edge (borda vermelha)) tiveram maior sensibilidade as variáveis florestais. Além disso, os resultados indicaram precisões de mapeamento aceitáveis, com raiz média do erro quadrático inferior a $80 \mathrm{~m}^{3} /$ ha. Devido a melhor resolução espacial, maior cobertura de área e resolução temporal, os autores sugerem o Sentinel-2/MSI para ser utilizado no mapeamento de volume de florestas aliado com a capacidade multiespectral do sensor EnMAP.

O estudo de Mura et al. (2018) avaliou o desempenho do Sentinel-2 para estimar o crescimento do volume de estoque de duas áreas florestais na Itália. Os dados do Sentinel-2/MSI foram comparados com Landsat-8/OLI e RapidEye. Os resultados indicaram que o Sentinel-2/MSI proporcionou estimativas melhores do que o Landsat- 8 em 37,5\% dos casos e em 62,5\% do que o RapidEye. O Landsat-8 foi superior em uma área de estudo, com desvio quadrado médio de 6,84\% e para a outra área, o Sentinel-2 apresentou o menor valor, com 22,94\%. Os autores concluíram que as imagens do Sentinel-2 são adequadas para a estimativa do crescimento do volume de áreas florestais.

Ao analisar os índices de vegetação oriundos dos dados do Sentinel-2, observou-se que o SAVI melhor se correlacionou com o volume. Esses resultados são consistentes com os obtidos por Alba et al. (2017) e Macedo et al. (2017) na estimativa volumétrica de florestas de eucalipto. Isso pode ser explicado pelo índice SAVI incorporar a resposta espectral do solo onde havia fechamento incompleto do dossel e pelas características geométricas na aquisição da imagem do sensor e pelo relevo plano da área, o qual pode ter influenciado na redução de efeitos negativos do contraste de iluminação entre os pontos amostrais (ALBA et al., 2017).

Vale a pena ressaltar as limitações existentes na associação entre dados remotamente situados e dados de inventário florestal. Meng et al. (2007) afirmam que a inclusão de características como geologia, clima, efeitos do solo e índice de sítio do povoamento no ajuste dos modelos favoreceria a precisão das estimativas. Outro detalhe importante diz respeito ao tamanho e a identificação das parcelas, pois nesse estudo, o tamanho das parcelas foram inferiores a resolução espacial do sensor

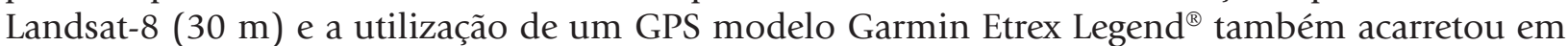
erros de localização em até 10 metros das parcelas em campo, ocasionados pelo fechamento do dossel florestal. Assim, deve-se ajustar modelos de regressão em função da área de estudo e do sensor utilizado.

Também é válido analisar as diferenças entre os dois sensores utilizados. Além da resolução espacial e espectral, a resposta espectral dos objetos, composição atmosférica e a curva de resposta espectral relativa de casa sensor apresentam diferenças (CHANDER et al., 2009). Esses fatores se relacionam diretamente com o brilho refletido e armazenado pelo sensor e podem acarretar em problemas em investigações que visam relacionar variáveis florestais e dados espectrais. 
Pertille et al. - Estimativa do volume de Pinus taeda L. a partir de dados de sensoriamento remoto

\section{CONCLUSÃO}

Para os dados do Landsat-8, o índice de vegetação que apresentou a melhor correlação com o volume foi o GNDVI e para o Sentinel-2, o SAVI.

Foi possível estimar o volume do povoamento através dos dados espectrais, porém, não houve diferença significativa entre o volume estimado pelo inventário florestal e por dados de Sensoriamento Remoto.

Recomenda-se que essa metodologia seja testada em outras espécies do gênero Pinus spp. e com sensores de média a alta resolução espacial.

\section{REFERÊNCIAS BIBLIOGRÁFICAS}

ALBA, E.; MARCHESAN, J.; MELLO, E.P.; TRAMONTINA, J.; SILVA, E.A.; PEREIRA, R.S. Uso de imagens de média resolução espacial para o monitoramento de dosseis de Eucalyptus grandis. Revista Scientia Agraria, Curitiba, v. 18, n. 4, p. 01-08, 2017.

ALVARES, C. A.; STAPE, J. L.; SENTELHAS, P.C.; GONÇALVES, J. L. M.; SPAROVEK, G. Köppen's climate classification map for Brazil. Meteorologische Zeitschrift, Stuttgart, v. 22, n. 6, p.711-728, 2013.

BERRA, E. F.; FONTANA, D. C.; KUPLICH, T. M. Comparison of satellite imagery from LISS-III/Resourcesat-1 and TM/ Landsat 5 to estimate stand-level timber volume. Pesquisa Florestal Brasileira, Colombo, v. 36, n. 88, p. 363-373, 2016.

CANAVESI, V.; PONZONI, F. J.; VALERIANO, M. M. Estimativa de volume de madeira em plantios de Eucalyptus spp. utilizando dados hiperespectrais e dados topográficos. Revista Árvore, Viçosa, v. 34, n. 3, p. 539-549, 2010.

CHANDER, G.; MEYER, D. J.; HELDER, D. L. Summary of current radiometric calibration coefficients for Landsat MSS, TM, ETM+, and EO-1 ALI sensors. Remote Sensing of Environment, New York, v. 113, n. 5, p. 893-903, 2009.

CHRYSAFIS, I.; MALLINIS, G.; GITAS, I. TSAKIRI-STRATI, M. Estimating Mediterranean forest parameters using multi seasonal Landsat 8 OLI imagery and an ensemble learning method. Remote Sensing of Environment, New York, v. 199, p.154-166, 2017.

ESRI - ENVIRONMENTAL SYSTEMS RESEARCH INSTITUTE. ArcGIS Professional GIS for the desktop, version 10.4.1. 2017. Disponível em: < https://support.esri.com/en/Products/Desktop/arcgis-desktop/arcmap/10-4-1 >. Acesso em: 20 dez. 2017.

GAO, B.C. NDWI a normalized difference water index for remote sensing of vegetation liquid water from space. Remote Sensing of Environment, New York, v. 58, p. 257-266, 1996.

GITELSON, A. A.; KAUFMAN, Y. J.; MERZLYAK, M. N. Use of a green channel in remote sensing of global vegetation from EOS-MODIS. Remote Sensing of Environment, New York, v. 58, p. 289-298. 1996.

HUETE, A. R. A soil-adjusted vegetation index (SAVI). Remote Sensing of Environment, New York, v. 25, n. 3, p. 295-309, 1988.

JENSEN, J.R. Sensoriamento remoto do ambiente: uma perspectiva em recursos terrestres. 2 ed. Tradução José Carlos Neves Epiphanio - São José dos Campos: Parêntese, 2009. 598 p.

JORDAN, C. F. Derivation of leaf-area index from quality of light on the forest floor. Ecology, v. 50, n. 406, p. 663-666, 1969.

LEAL, F. A.; MATRICARDI, E. A. T.; OLIVEIRA, K. A.; ALMEIDA, G. S. Índice de vegetação na estimativa do volume em um povoamento de eucalipto. Enciclopédia Biosfera, Goiânia, v. 9, n. 17, p.1638-1646, 2013.

MACEDO, F. L.; SOUSA, A. M. O.; GONÇALVES, A. C.; SILVA, H. R.; RODRIGUES, R. A. F. Estimativa do volume de madeira para Eucalyptus sp. com imagens de satélite de alta resolução espacial. Scientia Forestalis, Piracicaba, v. 45, n. 114, p. 237-247, 2017.

MENG, Q.; CEISZEWSKI, C. J.; MADDEN, M.; BORDES, B. A linear mixed-effects model of biomass and volume of trees using Landsat ETM+ images. Forest Ecology and Management, Amsterdam, v. 244, n. 3, p. 93-101, 2007. 
MURA, M.; BOTTALICO, F.; GIANNETTI, F.; BERTANI, R.; GIANNINI, R.; MANCINI, M.; ORLANDINI, S.; TRAVAGLINI, D.; CHIRICI, G. Exploiting the capabilities of the Sentinel-2 multi spectral instrument for predicting growing stock volume in forest ecosystems. International Journal of Applied Earth Observation and Geoinformation, v. 66, p.126-134, 2018.

NICOLETTI, M. F.; SOUZA, K.; SILVESTRE, R.; FRANÇA, M. C.; ROLIM, F. A. Relação Hipsométrica para Pinus taeda L. em Diferentes Fases do Ciclo de Corte. Floresta e Ambiente, Seropédica, v. 23, n. 1, p. 80-89, 2016.

NINK, S.; HILL, J.; BUDDENBAUM, H.; STOFFELS, J.; SACHTLEBER, T.; LANGSHAUSEN, J. Assessing the Suitability of Future Multi and Hyperspectral Satellite Systems for Mapping the Spatial Distribution of Norway Spruce Timber Volume. Remote Sensing, v. 7, p. 12009-12040, 2015.

R CORE TEAM. R: A language and environment for statistical computing. Vienna: R Foundation for Statistical Computing. Disponível em: < https://www.R-project.org/ >. Acesso em: 16 fev. 2018.

REUVENI, Y.; DAHAN, E.; ANKER, Y.; SPRINTSIN, M. Estimating forest parameteres using Landsat ETM+ spectral responses and monocultured plantation fieldwork measurements data. International Journal of Remote Sensing, London, v. 39, n. 8, p. 2620-2636, 2018.

SANQUETTA, C. R.; BALBINOT, R. Métodos de determinação de biomassa florestal. In: SANQUETTA, C. R.; BALBINOT, R.; ZILIOTTO, M. A. B. Fixação de carbono: atualidades, projetos e pesquisas. Curitiba: Ed. UFPR, 2004, 205 p.

SANTOS, M. M.; MACHADO, I. E. S.; CARVALHO, E. V.; VIOLA, M. R.; GIONGO, M. Estimativa de parâmetros florestais em área de Cerrado a partir de imagens do sensor OLI Landsat-8. Revista Floresta, Curitiba, v. 47, n. 1, p. 75-83, 2017.

SOUSA, A. M. O.; GOLÇALVES, A. C.; MESQUITA, P.; SILVA, J. R M. Biomass estimation with high resolution satellite images: A case study of Quercus rotundifolia. ISPRS Journal of Photogrammetry and Remote Sensing, Enschede, v. 101, p. 69-79, 2015.

SPANNER, M. A.; PIERCE, L. L.; PETERSON, D. L.; RUNNING, S. W. Remote Sensing temperate coniferous forest leaf area index: the influence of canopy closure, understory and background reflectance. International Journal of Remote Sensing, London, v. 11, n. 1, p. 95-111, 1990.

TESFAMICHAEL, S.; VAN AARDT, J.; AHMED, F. Estimating plot-level tree height and volume of Eucalyptus grandis plantations using small-footprint, discrete return LiDAR data. Progress in Physical Geography. v. 34, n. 4, p. 515-540, 2010.

Recebido em: 14/06/2018

Aceito em: 12/10/2018 